\title{
Liver condition and blood biochemical value of sturgeon fish when fed combined feed with increased fat content
}

\author{
D.V. Osepchuk ${ }^{1}$, N.A. Yurina ${ }^{1}$, D.A. Yurin ${ }^{1}$, E.V. Kuzminova ${ }^{1}$, M.P. \\ Semenenko ${ }^{1}$, A.N. Gneush ${ }^{1,2}$, E.A. Maksim ${ }^{1}$, A.A. Danilova ${ }^{1}$
}

\begin{abstract}
${ }^{1}$ Krasnodar Research Centre for Animal Husbandry and Veterinary Medicine, 4 Pervomayskaya Street, Znamensky, Krasnodar, 350055; ${ }^{2}$ Kuban State Agrarian University named after I. T. Trubilin, 13 Kalinin Street, Krasnodar, 350044, Russian Federation

*Corresponding author e-mail id: $\underline{4806144 @ \text { mail.ru }}$
\end{abstract}

Journal of Livestock Science (ISSN online 2277-6214) 12: 312-316

Received on 18/8/21; Accepted on 11/10/21; Published on 1/11/21

doi. 10.33259/JLivestSci.2021.312-316

\begin{abstract}
The results of the present experiment revealed that by feeding feed with a high fat content, the final weight of fish increases by $10.0-23.9 \%$, feed cost per $\mathrm{kg}$ of weight gain is reduced by $14.4-28.7 \%$. The condition factor of juvenile Russian-Lena sturgeon (hybrid of Acipenser baeri and Acipenser gueldenstaedtii) increases by 12.6-31.6\%. With an increase in the percentage of feeding fat-rich feed, an increase in liver mass indices by $13.9-17.8 \%$, spleen mass indices by $13.0 \%$, a decrease in intestinal mass indices in fish of the third and fourth groups - by 15.4 and $17.1 \%$, and stomach of juveniles - by 7.4 and $9.0 \%$. An increase in the severity of fatty hepatosis associated with a certain level of feeding affected the carbohydrate metabolism of fish. In the experimental groups (with a fat content of 15.8-20.0\% in compound feed), there is a higher glucose content in relation to the control group (with a fat content of $14 \%$ in compound feed), with a difference of $12.7-13.5 \%$. The biochemical picture indicates the liver damage in the fish of the experimental groups, while during the transition to a more pronounced stage, the cholesterol concentration falls below normal. An increase in AST activity was - by 14.0-60.7\%, relative to the control. In the level of ALT, an increase in the indicator was recorded only in the second and third experimental groups with a difference of $12.1 \%$ and $14.1 \%$, respectively, and in the fourth experimental group, on the contrary, the content of this enzyme was lower by $29.4 \%$ relative to the control group. Considering the significant degree of liver damage in fish of this group, this can be explained by the fact that the peak of destruction of hepatocytes has already passed, and then the level of aminotransferases becomes minimal.
\end{abstract}

Keywords: fatty hepatosis; feeding; sturgeon; weight gain; the condition factor. 


\section{Introduction}

Industrial breeding of valuable fish species to obtain quality products for human nutrition is attracting more and more attention (Józefiak et al, 2019, Xu et al, 2019, Geraylou et al, 2012). However, the intensification of the aquaculture industry gave rise to the emergence and spread of non-infectious diseases of sturgeon fish. The affection can also be caused by disease associated with malnutrition and, as a result, metabolism, for example, fatty degeneration of the liver (Loponte et al, 2017, Mikoiajczak et al, 2020, Rapatsa et al, 2019, Abdel-Warith et al, 2020).

Due to its high functional specialization, the liver has a special place in maintaining the metabolism of the fish organism. At the same time, the use of highly concentrated mixed fodders with an increased level of easily oxidizable lipids and a decrease in the degree of phospholipid unsaturation lead to a deterioration in liver function (Kryuchkov et al, 2006, Kryuchkov et al, 2007, Dengyue et al, 2019, Lee et al, 2017, Hung, 2017).

The fish liver performs the main load under conditions of increased technogenic pressure, and the survival of the organism and its functions directly depend on its functional activity. The overwhelming majority of fish diseases are associated with keeping and feeding conditions. The treatment of fish diseases associated with metabolic disorders and decreased liver function consists in their high-quality prevention through more careful balancing of compound feed and feeding hepatoprotectors (Wei et al, 2020, Falah et al., 2020, Yousefi et al, 2020).

Objective of the research: is to assess the state of the liver of sturgeon fish when consuming compound feeds with a high fat content, taking into account their biochemical blood parameters, as well as the features of the structure and function of the liver.

To achieve the stated goal, the following tasks were solved:

1. The main fish-breeding indicators were determined when feeding feeds with a high fat content.

2. The morphometric analysis of fish was carried out.

3. The methods of cluster analysis revealed the differences between healthy individuals and those with fatty liver in terms of the content of a number of biochemical blood parameters: total protein, urea, cholesterol, glucose, aminotransferase - AST and ALT, direct and total bilirubin.

On the basis of the data obtained, a lifetime assessment of the state of the liver of the fish organism will be developed according to a set of signs and indicators.

\section{Material and methods}

As a result of the study of a sample of pond water in fish tanks for keeping sturgeon fish at Albashi LLC, it was revealed that nitrates and nitrites are present within the permissible limits. The ammonia content is less than $0.04 \mathrm{mg}$ (N) $/ \mathrm{dm}^{3}$, which does not exceed the MPC. When measuring the $\mathrm{pH}$, it was found that the reaction of the medium is weakly alkaline, and is within the permissible values. Biological oxygen demand $\left(\mathrm{BOD}_{5}\right)$ is $1.4 \mathrm{mg}\left(\mathrm{O}_{2}\right) / \mathrm{dm}^{3}$, which did not exceed the established standards, and indicates that the amount of easily oxidized organic pollutants in water is within acceptable limits. The amount of oxygen dissolved in water is $10.6 \mathrm{mg} / \mathrm{dm}^{3}$, which corresponds to the standards for fishery reservoirs.

The content of suspended solids, total iron, sulfates, phosphates, chlorides did not exceed the established norms. Thus, in order to identify a factor of possible intoxication, it is necessary to regularly monitor the hydrochemical parameters during fish farming.

Scientific research was carried out at the fish farm LLC "Albashi" of the Leningradsky district of the Krasnodar Territory. Albashi LLC has channel ponds with a total area of 2 and 35 hectares and a dug pond with a total area of 1 hectare, where carp, silver carp and sazan are grown. There are two fish-breeding modules for the basin keeping of sturgeon fish of all age and sex groups, a department for incubating fish eggs, cages in a channel pond for keeping large fish.

In the farm where the research was carried out, no signs of hepatosis were found in fish in the first half of the year. Therefore, it was decided to artificially induce hepatosis of fish when feeding fodder enriched with fat. In order to obtain a picture of the manifestation of hepatosis and to associate them with the appearance of a macular spot on the abdomen of fish, in the scientific experiment, the juvenile of the experimental groups was given trout food with a higher fat content than in the complete sturgeon compound feed.

The experiment used juvenile of Russian-Lena sturgeon (underyearlings). The experiment was carried out according to the following scheme: the first group of juveniles was given a complete sturgeon compound feed (Sturgeon $\mathrm{CF}$ ) with a granule size of $3 \mathrm{~mm}$, produced by Aller Trident EX with a protein content of $47.0 \%$, fat - 14.0\%, metabolizable energy (ME) - 21.1 MJ. The second group received Sturgeon CF $+30 \%$ trout PC Aller Silver (the protein content with this combination in $100 \mathrm{~g}$ was: $46.4 \%$, fat $-15.8 \%$, ME $-21.5 \mathrm{MJ}$ ); the third group - Sturgeon $\mathrm{CF}+50 \%$ trout CF (protein $-46.0 \%$, fat $-17.0 \%$, ME - $21.7 \mathrm{MJ}$ ); the fourth group received Trout CF (protein - $45.0 \%$, fat $20.0 \%$, ME - 22.3 MJ). The experiment lasted 90 days.

Aller Trident EX feed ingredients: wheat, wheat gluten, rapeseed oil, fish oil, fishmeal, soy protein, soy, yeast, corn gluten, sunflower protein, vitamins, premix and mineral supplements.

Aller Silver feed ingredients: fishmeal, fish oil, soy meal, hemoglobin meal, hydrolyzed protein, rapeseed, wheat, vitamins and mineral supplements.

The main indicators characterizing the growth rate of juvenile fish are its mass and feed costs per unit of 
weight gain (feeding ratio). Sturgeon fry were weighed individually on an electronic balance at the beginning and end of the experiment. Gross and average daily gains for experiment were calculated. The length of the fish was measured from the top of the snout to the vertical of the end of the longest blade of the caudal fin with the fish in a horizontal position. Body condition coefficient (C) was calculated as the ratio of weight to body length according to T. Fulton's formula. Morphometric analysis was performed on 6 fish specimens in order to study the state of the liver and other internal organs. The studies were carried out according to the method of conducting experiments on feeding fish described in the book by Sklyarova (2008).

Blood was collected for analysis during morphometric analysis. Blood biochemical parameters were determined on an automated biochemical analyzer - Vitalab Selectra Junior with software version 1.0. (an open system for photometric tests, manufactured by Vital Scientific N. V. Netherlands) using reagents from ELITech Clinical Systems (France) and Analyticon biotechnologies AG (Germany).

\section{Results}

The main fish-breeding indices of the rearing of juvenile Russian-Lena sturgeon were obtained as follows: the initial mass of fish when they were placed in the basins was the same. However, at the end of the growing period, significant differences were observed. The final weight of sturgeon in the second group increased significantly (P $<0.001$ ) by $10.0 \%$, in the third - by $23.9 \%$, in the fourth - by $13.3 \%$.

The consumption of food in all groups was the same, since the feeding was carried out in a standardized manner, and amounted to $400 \mathrm{~g}$ for the entire period of the experiment per 1 fish. However, feed costs (feeding ratio) per $1 \mathrm{~kg}$ of live weight gain were lower in the experimental groups by $14.4 \%, 28.7 \%$ and $18.2 \%$, respectively.

The expenditures of metabolizable energy per $1 \mathrm{~kg}$ of fish weight gain in the control group were $0.38 \mathrm{MJ}$, in the second experimental group - by $13.2 \%$ lower, in the third group - by $26.3 \%$ and in the fourth - by $13.2 \%$ lower. Protein consumption per $1 \mathrm{~kg}$ of live weight gain was lower than the control indicator by $15.3,30.6$ and $21.2 \%$, respectively, in groups. The costs of fat per $1 \mathrm{~kg}$ of fish weight gain was $12.0 \%$ lower in the third experimental group and $16.0 \%$ higher in the fourth experimental group, compared with the control.

The condition factor of juvenile Russian-Lena sturgeon, calculated according to Fulton, was higher in the second group - by $12.6 \%$, in the third group - by $31.6 \%$ and in the fourth group - by $26.2 \%$, in comparison with the control.

The results obtained allow us to conclude that the use of trout feed in feeding juvenile Russian-Lena sturgeon in the basins contributes to an increase in the growth rate of fish. It would seem, it was worth recommending it for use to increase the growth rate of fish, but it is necessary to monitor the condition of the fish for obesity of liver tissue and further forecast of its cultivation.

When carrying out a morphometric analysis of fish $(n=6)$, it was found that in the first group the fish liver had a rich red colour and a dense consistency. In the second group, the indicators were in an intermediate state. In the third and fourth groups, hepatosis was visually expressed. The liver of the fish of the third and fourth groups was enlarged, had a yellowish and creamy colour, and a looser consistency. At the same time, there was an increase in liver indices in the experimental groups: 1.01 was in the control group, in the second -1.08 , in the third -1.15 , or higher than the control by $13.9 \%(\mathrm{P}<0.001)$, in the fourth -1.19 , or $17.8 \%$ higher than the control $(\mathrm{P}<0.001)$. With hepatosis, there is an increase in the spleen mass, which manifested itself in the experimental groups, the difference in the spleen index in the third and fourth experimental groups was $13.0 \%$ higher $(\mathrm{P}<0.05)$ relative to the control.

The heart of fish consists of four chambers: the sinus venous, where venous blood is collected; atrium; ventricle and conus arteriosus. There was a slight increase in heart mass indices in the experimental groups by 3.6-7.1\% relative to the control, however, there was no significant difference, but the tendency towards cardiac obesity was obvious.

The presence of a large amount of fat in the feed causes a faster passage of chyme through the gastrointestinal tract of fish, which contributed to a decrease in intestinal mass indices in fish of the third and fourth groups by 15.4 and $17.1 \%(\mathrm{P}<0.001)$ and the stomach of juveniles - by 7.4 and $9.0 \%(\mathrm{P}<0.01)$.

It was found that the surface of the gills, which are respiratory organs, are compact and highly vascularized. Four well-developed branchial arches are located under the operculum. On each branchial arch, on the side facing the oral cavity, there are branchial stamens, which serve to retain food lumps. Opposite them, there are the gill lobes that perform the respiratory function. The branchial lobes of the adjacent branchial arches fit tightly to each other, thereby forming the branchial lattice through which water passes. In the fish of all groups, no pathology was recorded in the state of the gills. No differences were found between the groups either.

The swim bladder of fish is a derivative of the intestine. In the Russian-Lena sturgeon, it has the shape of a bag of white-silver colour, located between the spine and intestines. No pathologies and differences between the groups in its development were found.

During the morphometric analysis of experimental sturgeon fish, their excretory system was also analyzed. The kidneys of all fish groups were deep dark red. They were located physiologically, in the body cavity under the spine on both sides of the dorsal artery. In all individuals, there were no pathologies recorded in their development.

A biochemical study of the blood of fish (Table 1) showed that in the second, third and fourth groups there was a lower level of total protein in relation to the first control group, in which the best indicators of protein metabolism 
were recorded. The difference in this indicator was: in the second group - 52.1\% (P<0.05); in the third group - 48.9\%; in the fourth group $-62.2 \%(\mathrm{p}<0.01)$. There is also an increase in the degree of violation of the protein-synthetic function of the liver, which is confirmed by a lower concentration of urea, with a difference from the control: in the second group - $2.2 \%$; in the third group - $18.6 \%$; in the fourth group $-29.4 \%(\mathrm{P}<0.05)$.

Table 1 - Biochemical parameters of fish blood $(\mathrm{M} \pm \mathrm{m} ; \mathrm{n}=5)$

\begin{tabular}{|l|c|c|c|c|}
\hline \multirow{2}{*}{\multicolumn{1}{c|}{ Parameters }} & \multicolumn{4}{c|}{ Group } \\
\cline { 2 - 5 } & 1 & 2 & 3 & 4 \\
\hline Total protein, g / 1 & $21.9 \pm 0.87$ & $14.4 \pm 0.74^{*}$ & $14.7 \pm 1.32$ & $13.5 \pm 0.63^{* *}$ \\
\hline Urea, mmol / 1 & $3.72 \pm 0.25$ & $3.62 \pm 0.21$ & $3.12 \pm 0.42$ & $2.86 \pm 0.16^{*}$ \\
\hline Glucose, $\mathrm{mmol} / \mathrm{l}$ & $2.95 \pm 0.13$ & $2.99 \pm 0.18$ & $3.38 \pm 0.40$ & $3.41 \pm 0.39$ \\
\hline AST U/1 & $216.8 \pm 21.6$ & $252.0 \pm 14.6$ & $317.6 \pm 22.8$ & $357.2 \pm 31.4$ \\
\hline ALT, U/l & $203.2 \pm 16.9$ & $231.2 \pm 21.6$ & $236.6 \pm 28.6$ & $157.0 \pm 34.0$ \\
\hline Cholesterol, $\mathrm{mmol} / \mathrm{l}$ & $2.66 \pm 0.15$ & $2.68 \pm 0.23$ & $2.81 \pm 0.12^{*}$ & $1.65 \pm 0.11^{* *}$ \\
\hline Total bilirubin, $\mu \mathrm{mol} / \mathrm{l}$ & $7.8 \pm 0.10$ & $7.9 \pm 0.11$ & $8.08 \pm 0.28$ & $8.1 \pm 0.34$ \\
\hline
\end{tabular}

Note: the differences are significant when $*$ - $\mathrm{P}<0.05 ; * *$ - $\mathrm{P}<0.01$ relative to the first group

An increase in the severity of fatty hepatosis associated with a certain level of feeding was reflected in the carbohydrate metabolism of fish. Thus, in the third and fourth experimental groups, there is a higher glucose content in relation to the control group, with a difference of $12.7 \%$ and $13.5 \%$, respectively. Perhaps, in this case, the development of fatty hepatosis occurs against the background of a decrease in the quality of the work of the pancreas, which provokes a steady rise in the level of glucose in the blood of fish.

Aspartate aminotransferase (AST) and alanine aminotransferase (ALT) are hepatoindicator enzymes; comparative assessment of these indicators in studies revealed an increase in AST activity - in the second group by $14.0 \%$, in the third group - by $31.7 \%$ and in the fourth group by $60,7 \%$ regarding the control. An increase in the ALT level was recorded only in the second and third experimental groups with a difference of $12.1 \%$ and $14.1 \%$, respectively, and in the fourth experimental group, on the contrary, the content of this enzyme was lower by $29.4 \%$ relative to the first group. Considering the significant degree of liver damage in the fish of this group, this can be explained by the fact that the peak of destruction of hepatocytes has already passed, and then the level of aminotransferases becomes minimal.

A similar situation between the groups is observed in the content of cholesterol, which is confirmed by its higher concentration in the third experimental group, relative to the control with a difference of 5.3\% (p<0.05). A similar biochemical picture indicates the initial degree of liver damage to the fish of the third group, while during the transition to a more pronounced stage, the cholesterol concentration falls below normal, which was revealed in the fourth experimental group, with a difference from the control by 1.6 times $(\mathrm{p}<0.01)$. The content of bilirubin in the blood tended to increase in the third and fourth experimental groups - by an average of $3.5 \%$.

\section{Discussion}

An increase in the severity of fatty hepatosis associated with a certain level of feeding affected the carbohydrate metabolism of fish. In the third and fourth experimental groups, there is a higher glucose content in relation to the control group, with a difference of $12.7-13.5 \%$. The biochemical picture indicates the initial degree of liver damage in the fish of the third group, while during the transition to a more pronounced stage in the fourth group, the cholesterol concentration falls below normal. An increase in AST activity was - by 14.0-60.7\%, relative to the control. In the level of ALT, an increase in the indicator was recorded only in the second and third experimental groups with a difference of $12.1 \%$ and $14.1 \%$, respectively, and in the fourth experimental group, on the contrary, the content of this enzyme was lower by $29.4 \%$ relative to the first group. Considering the significant degree of liver damage in fish of this group, this can be explained by the fact that the peak of destruction of hepatocytes has already passed, and then the level of aminotransferases becomes minimal.

The results of our research are consistent with with the results of similar research work done in the past by Mikoiajczak et al, 2020.

\section{Conclusion}

It was found that when feeding trout feed with a high fat content in the composition of $\mathrm{CF}$, the final weight of fish increases by $10.0-23.9 \%$. Feed costs per $1 \mathrm{~kg}$ of weight gain are reduced by $14.4-28.7 \%$. The condition factor of juvenile Russian-Lena sturgeon increases by 12.6-31.6\%. With an increase in the percentage of feeding fat-rich feed, an increase in liver mass indices by $13.9-17.8 \%$, spleen mass indices by $13.0 \%$, a decrease in intestinal mass indices in fish of the third and fourth groups - by 15.4 and $17.1 \%$, and stomach of juveniles - by 7.4 and $9.0 \%$. 


\section{Acknowledgement}

The research was carried out within the framework of the grant of the President of the Russian Federation for state support of young Russian scientists on the topic: "Development of criteria for assessing the state of the liver of sturgeon fish" MD-1439.2020.11.

\section{References}

1) Abdel-Warith A.A., Younis E.M., Al-Asgah N.A., Mahboob S. Braz J. 2020. Biol. Effect of replacing fish meal by full fat soybean meal on growth performance, feed utilization and gastrointestinal enzymes in diets for African catfish Clarias gariepinus. 80(3), 535-543. doi: 10.1590/1519-6984.214763.

2) Dengyue Y., Yundi G., Xin Z., Bin W. et al. 2019. NPY and NPY receptors in the central control of feeding and interactions with CART and MC4R in Siberian sturgeon. General and Comparative Endocrinolog. 284 (1), 113239. doi: 10.1016/j.ygcen.2019.113239.

3) Falah F.J., Islami H.R., Mehrgan M.S. 2020. Dietary folic acid improved growth performance, immuno-physiological response and antioxidant status of fingerling Siberian sturgeon, Acipenser baerii (Brandt 1896). Aquaculture Reports. 17, 100391, doi: doi.org/10.1016/j.aqrep.2020.100391.

4) Geraylou Z., Souffreau C., Rurangwa E., D'Hondt S., Callewaert L., Courtin C.M., Delcour J.A., Buyse J., Ollevier F. 2012. Effects of arabinoxylan-oligosaccharides (AXOS) on juvenile Siberian sturgeon (Acipenser baerii) performance, immune responses and gastrointestinal microbial community. Fish Shellfish Immunol. 33(4), 718-24. doi: 10.1016/j.fsi.2012.06.010.

5) Hung S.S.O. 2017. Recent advances in sturgeon nutrition. Animal Nutrition. Vol. 3(3), 191-204. doi: 10.1016/j.aninu.2017.05.005

6) Józefiak A., Nogales-Mérida S., Rawski M., Kierończyk B., Mazurkiewicz J. 2019. Effects of insect diets on the gastrointestinal tract health and growth performance of Siberian sturgeon (Acipenser baerii Brandt, 1869) BMC Vet Res. 15(1), 348. doi: 10.1186/s12917-019-2070-y.

7) Kryuchkov V.N., Dubovskaya A.V., Fomin I.V. 2006. Issues of ecological physiology of fish. / Bulletin of ASTU. 3 (32), 94-100.

8) Kryuchkov V.N., Shaydullina Zh.M. 2007. Morphological and biochemical analysis of the liver of the bream of the Ural River. South of Russia: ecology, development. 3, 57-59.

9) Lee S., Sonmez O., Hung S.S.O., Fadel J.G. 2017. Development of growth rate, body lipid, moisture, and energy models for white sturgeon (Acipenser transmontanus) fed at various feeding rates. Animal Nutrition. Vol. 3(1), pp 46-60. doi: 10.1016/j.aninu.2016.10.005

10) Loponte R., Nizza S., Bovera F., De Riu N., Fliegerova K., Lombardi P., Vassalotti G., Mastellone V., Nizza A., Moniello G. 2017. Growth performance, blood profiles and carcass traits of Barbary partridge (Alectoris barbara) fed two different insect larvae meals (Tenebrio molitor and Hermetia illucens). Res Vet Sci. 115, 183188. doi: 10.1016/j.rvsc.2017.04.017.

11) Mikoiajczak Z., Rawski M., Mazurkiewicz J., Kierocczyk B., Jyzefiak D. 2020. The Effect of Hydrolyzed Insect Meals in Sea Trout Fingerling (Salmo trutta m. trutta) Diets on Growth Performance, Microbiota and Biochemical Blood Parameters. Animals (Basel). 10(6), 1031. doi: 10.3390/ani10061031.

12) Rapatsa M.M., Moyo N.A.G. 2019. Enzyme activity and histological analysis of Clarias gariepinus fed on Imbrasia belina meal used for partial replacement of fishmeal. Fish Physiol Biochem. 45(4), 1309-1320. doi: 10.1007/s10695-019-00652-3.

13) Wei H.C., Xing S.J., Chen P., Wu X.F. et al. 2020. Plant protein diet-induced hypoimmunity by affecting the spiral valve intestinal microbiota and bile acid enterohepatic circulation in Amur sturgeon (Acipenser schrenckii). Fish \& Shellfish Immunology. 106, 421-430. doi: 10.1016/j.fsi.2020.08.025

14) Xu G., Xing W., Li T., Xue M., Ma Z., Jiang N., Luo L. 2019. Comparative study on the effects of different feeding habits and diets on intestinal microbiota in Acipenser baeri Brandt and Huso huso. BMC Microbiol.19(1), 297. doi: 10.1186/s12866-019-1673-6.

15) Yousefi S., Shokri M.M., Noveirian H.A., Hoseinifar S.H. 2020. Effects of dietary yeast cell wall on biochemical indices, serum and skin mucus immune responses, oxidative status and resistance against Aeromonas hydrophila in juvenile Persian sturgeon (Acipenser persicus). Fish \& Shellfish Immunology. Vol. 106, pp 464472. doi: 10.1016/j.fsi.2020.08.007 